\title{
MET deletion is a frequent event in gastric/gastroesophageal junction/esophageal cancer: a cross-sectional analysis of gene status and signal distribution in 1,580 patients
}

\author{
Jan Trøst Jørgensen ${ }^{1}$, Jens Mollerup ${ }^{2}$, Hui Yang ${ }^{3}$, Ning Go $^{3}$, Karsten Bork Nielsen ${ }^{4}$ \\ ${ }^{1}$ Companion Diagnostics, Dx-Rx Institute, Fredensborg, Denmark; ${ }^{2}$ Pathology Division, Agilent Technologies, Glostrup, Denmark; ${ }^{3}$ Medical \\ Sciences, Amgen Inc., Thousand Oaks, USA; ${ }^{4}$ Bork Enterprises, Virum, Denmark \\ Contributions: (I) Conception and design: N Go, KB Nielsen, JT Jørgensen; (II) Administrative support: All authors; (III) Provision of study materials \\ or patients: N Go, KB Nielsen, JT Jørgensen; (IV) Collection and assembly of data: H Yang, J Mollerup, JT Jørgensen; (V) Data analysis and \\ interpretation: All authors; (VI) Manuscript writing: All authors; (VII) Final approval of manuscript: All authors. \\ Correspondence to: Dr. Jan Trøst Jørgensen. Companion Diagnostics, Dx-Rx Institute, Baunevaenget 76, DK-3480 Fredensborg, Denmark. \\ Email: jan.trost@dx-rx.dk.
}

Background: MET gene aberrations are found in several human cancers including gastric, ovarian and lung. In a large multinational cohort of patients with gastric/gastroesophageal junction/esophageal (G/GEJ/ E) adenocarcinoma we assessed the MET status with respect to amplification and deletion and correlate the results with the phenotypical gene signal distribution pattern.

Methods: Tissue specimens from 1,580 patients were analyzed using a novel fluorescence in situ hybridization (FISH) assay employing a MET/CEN-7 IQFISH Probe Mix. MET amplification and deletions were defined as a $M E T / C E N-7$ ratio $\geq 2.0$ and a $M E T / C E N-7$ ratio $<0.8$, respectively. Furthermore, the link between the MET gene status and the phenotypical signal distribution was investigated.

Results: The prevalence of $M E T$ amplification and deletions was found to be $7.2 \%$ and $8.7 \%$, respectively. Significant differences were observed with regard to geographic regions and sex. The Asian population had the highest percentage of MET amplification (9.4\%) and the lowest percentage of deletions (3.2\%). MET deletions was found more frequently among males (10.1\%) compared to females $(5.3 \%)$ and in esophagus (17.6\%) compared to the stomach (5.7\%). More than $50 \%$ of the patients who harbored MET gene amplification had a heterogeneous distribution of the FISH signals. Patients with a focal signal distribution were solely to be found among the MET amplified population. MET deletion were mainly observed in the group of patients with a homogenous signal distribution.

Conclusions: The screening data from this cross-sectional study showed that MET deletion and amplification are frequent events in G/GEJ/E cancer, which are linked to different phenotypical signal distribution patterns. The role of $M E T$ deletion in relation to tumor development is not fully understood but it is likely to play a role in the oncogenic transformation of the cells.

Keywords: Mesenchymal epithelial transition factor gene (MET); fluorescence in situ hybridization (FISH); gastric cancer; amplification; deletion

Submitted May 19, 2020. Accepted for publication Nov 01, 2020.

doi: 10.21037/atm-20-4081

View this article at: http://dx.doi.org/10.21037/atm-20-4081

\footnotetext{
$\wedge$ ORCID: 0000-0001-6255-0006.
} 


\section{Introduction}

The mesenchymal epithelial transition factor gene (MET) is a proto-oncogene located on chromosome $7 \mathrm{q} 31$ that encodes a transmembrane receptor with intrinsic tyrosine kinase activity known as Met (or cellular-MET, c-Met) (1). This receptor is also called the hepatocyte growth factor receptor (HGFR) after its ligand; hepatocyte growth factor (HGF) (1-3). Synthesis of HGF by neighboring cells and binding to Met results in tyrosine phosphorylation and activation of the Met receptor. During non-tumorigenic conditions, the paracrine activation of Met has key roles in embryogenesis, wound healing and organ regeneration (4). In cancer patients, aberrant Met signaling can result in suppression of apoptosis and cell proliferation, motility, migration, and invasion, which likely arises from different genetic alterations or dysregulation of MET (3-6). These genetic alterations also include amplification of $M E T$, which has been found in several human cancers, including gastric, ovarian, lung, breast, renal and more (6).

In studies of gastric/gastroesophageal junction/ esophageal (G/GEJ/E) cancer, MET amplification has been reported with a prevalence in the range of $1.5 \%$ to $30.5 \%$, depending on the study and the analytical method used (7-18). Using the in situ hybridization (ISH) techniques, such as fluorescence in situ hybridization (FISH), silver in situ hybridization (SISH), or chromogenic in situ hybridization (CISH), a prevalence in the range of $1.5 \%$ to $8.3 \%$ has been reported (7-15). MET amplification can also be detected using real-time polymerase chain reaction (qPCR); however, the prevalence seems to be somewhat higher than with the different ISH techniques with published data in the range of $3.7 \%$ to $30.5 \%$ (16-19). The results from these two analytical methods are not directly comparable due to differences in the way the assays detect gene amplification. The qPCR-based assays identify a gain in gene copy number regardless of the underlying cause and are thus unable to discriminate gene amplification from polysomy (6). This discrimination seems to be important as true $M E T$ amplification is more likely to lead to oncogenic MET addiction than polysomy (20). Furthermore, in a recent comparison of $M E T$ amplifications in solid tumors by in situ and extraction-based methods, large discrepancies was found compared with extraction-based methods such as PCR and NGS (21). Another factor contributing to the variability is the differences in the cut-off selected for the individual assays, thus comparing prevalence data for MET gene amplification across different study populations can be challenging (9). Recently, in a review by Guo et al., it is likewise diligently described how MET amplification may vary with the technique or assay used (20). Even, comparing results from different ISH assays can be difficult due to the differences in the scoring algorithm used (7-15). When it comes to deletion of the MET gene in patients with G/GEJ/E cancer, far less is known both with respect to the prevalence as well as how this aberration influences the disease processes (22).

Several studies in patients with G/GEJ/E cancer have reported that $M E T$ amplification is associated with an unfavorable clinical outcome, which has been shown from data generated both with the ISH and qPCR assays $(8,10,16,17,19)$. The association between MET amplification and a poor disease prognosis has likewise been confirmed in a meta-analysis (23). Beside the disease prognostic characteristics, it has been suggested that MET amplification potentially possesses predictive properties in relation to Met-targeted therapy and, thereby could act as a companion or complementary diagnostic in relation to the tyrosine kinase inhibitors under development for treatment of G/GEJ/E cancer and other indications (13,15,24-26). Available data suggest that treatment plans targeting both Met and Her2 (human epidermal growth factor receptor 2 gene product) may be beneficial. For example, patients with HER2 amplified trastuzumab-resistant esophageal cancer showed that the effect of therapy with afatinib correlated with MET co-amplification (27). Furthermore, resistance towards afatinib in a gastric cancer cell line was reversed by MET knockdown indicating MET amplification as a resistance factor towards afatinib treatment (28).

Recent studies in patients with G/GEJ/E adenocarcinoma have suggested a link between gene amplification and a heterogeneous signal distribution pattern. This link has been shown for both the HER2 and MET genes $(9,29)$. Here, we report data from a large cross-sectional study on $M E T$ gene aberrations in patients with G/GEJ/E adenocarcinoma analyzed with a FISH assay using the formamide-free, fast IQFISH hybridization buffer $(30,31)$. The purpose of this exploratory study was to investigate the prevalence of MET amplification and deletions in tissue specimens from a large cohort of patients with G/GEJ/E adenocarcinoma and correlate these findings with the phenotypical gene signal distribution pattern. We present the following article in accordance with the STREGA reporting checklist (available at http://dx.doi.org/10.21037/ atm-20-4081). 


\section{Methods}

\section{Patients/specimens}

The study included 1,580 formalin-fixed and paraffinembedded (FFPE) G/GEJ/E adenocarcinoma specimens consecutively collected from the screening population of an international multi-center phase II trial with the Met tyrosine kinase inhibitor AMG337 (Amgen) (25). The limited demographic and clinical data were collected in relation with the $M E T$ eligibility testing for inclusion in the clinical phase II study with AMG337. The G/GEJ/E specimens were cut in sections of $4 \mu \mathrm{m}$ and mounted on glass slides. Apart from data on the MET gene status and the signal distribution pattern no other demographic and clinicopathological data were available except sex, age and tumor site. The study was conducted according to the Declaration of Helsinki (as revised in 2013), and informed consent was received from patients prior to testing of the biopsy specimens. Prior to study initiation the protocol was reviewed and approved by Institutional Review Boards/Ethics Committees in the respective countries. The study was conducted under a US Investigational New Drug Application and Investigational Device Exemption according to the International Conference on Harmonization (ICH) Good Clinical Practice (GCP) guideline (ClinicalTrials.gov ID: NCT02016534/EudraCT Number: 2013-001277-24). The study was conducted at 97 clinical sites worldwide. Evaluation of the stained slides was performed at one central laboratory in the USA by a technologist and subsequently independently reviewed by a pathologist.

\section{MET IQFISH Testing}

FISH staining using the MET/CEN-7 IQFISH Probe Mix (For Investigational Use Only (IUO), Dako/Agilent Technologies) and reagents in the Histology FISH Accessory Kit (Dako/Agilent Technologies) was performed according to the IQFISH staining procedure (9). Following mounting of the FFPE specimens on positively charged glass slides, heat pre-treatment was carried out in a microwave oven. Then pepsin digestion was done at $37^{\circ} \mathrm{C}$ followed by ethanol dehydration with subsequent drying prior to probe application. The Texas Red-labeled DNA probe $(M E T)$ and the fluorescein-labeled PNA probe (CEN-7) were co-denatured with the tissue specimen for 10 minutes at $66^{\circ} \mathrm{C}$ followed by hybridization at $45^{\circ} \mathrm{C}$ for 90 minutes using a Hybridizer (Dako/Agilent Technologies).
Following hybridization, excess probe was washed away with Stringent Wash Buffer at $63{ }^{\circ} \mathrm{C}$ for 10 minutes. Then the slides were dehydrated in ethanol baths, dried at room temperature and, finally, the slides were mounted in DAPIcontaining Fluorescence Mounting Medium with glass coverslips.

MET FISH stained slides were inspected using a fluorescence microscope equipped with $20 x, 40 x$ and $100 x$ objectives and enumeration was performed at the largest magnification. Ratios of MET/CEN-7 from the invasive tumor area were calculated by counting of signals from 20 nuclei. Based on this ratio, specimens were characterized as amplified (MET/CEN-7 $\geq 2.0$ ), non-amplified (MET/ CEN-7 between $\geq 0.8$ and 2.0), or deletion (MET/CEN-7 $<0.8$ ). Specimens having a ratio between 1.8 and 2.2 were considered borderline cases and signals from additional 20 nuclei were counted and the final ratio and MET status was determined from the 40 nuclei. The cut-off level for $M E T$ deletion was selected based on previous observations for other genes using FISH assays (32-34). Signals in normal cells within the sample were used to verify staining quality of individual specimens, as these cells are expected to have a normal diploid status with two MET gene signals and two CEN-7 signals in every nucleus. Before the MET FISH assay was used in the study, it underwent an extensive analytical validation (9).

\section{MET signal distribution}

In addition to the assessment of $M E T$ gene status (amplified/ non-amplified/deletion), the $M E T$ signal distribution pattern was evaluated. In these tumors, $M E T$ gene signals can preferentially have a homogeneous or heterogeneous distribution pattern (9). The homogeneous signal distribution is observed when most tumor cells in the tumor specimen are equally amplified or equally non-amplified. The heterogeneous signal distribution is observed when tumor cells having amplified and non-amplified status are intermingled across the tumor. Specimens with the heterogeneous signal distribution are further divided into two categories: (I) When MET amplified tumor cells are grouped together, the signal distribution is categorized as focal, and, (II) when MET amplified tumor cells are interspersed in tissue areas of cells exhibiting low or normal $M E T / C E N-7$ ratio, the signal distribution is defined as mosaic (9). A similar phenotypical signal distribution pattern has been described for HER2, another gene known to be amplified in a subset of patients with G/GEJ/E cancer (29). 
Table 1 Demographic and tumor characteristics of the G/GEJ/E cancer patients screened for $M E T$ amplification $(\mathrm{N}=1,580)$

\begin{tabular}{lc}
\hline Characteristics & $\mathrm{N}(\%)$ \\
\hline Age, years & $60.3(12.1)$ \\
Mean (SD) & $62.0(19-96)$ \\
Median (Range) & \\
Regions & $277(17.5)$ \\
Asia $^{1}$ & $1,142(72.3)$ \\
Europe/Australia & \\
North America & \\
South America & \\
Sex & $154(9.7)$ \\
Male & $7(0.4)$ \\
Female & $1,132(71.6)$ \\
Tumor site & $448(28.4)$ \\
Gastric & \\
Gastroesophageal Junction & \\
Esophageal & $981(62.1)$ \\
Metastatic & $169(10.7)$ \\
\hline
\end{tabular}

${ }^{1}$ Republic of Korea; ${ }^{2}$ Australia, Belgium, Czech Republic, France, Germany, Greece, Hungary, Italy, Poland, Russian Federation, Spain, United Kingdom; ${ }^{3}$ Canada, United States of America; ${ }^{4}$ Chile, Peru; G/GEJ/E, gastric/gastroesophageal junction/ esophageal; MET, mesenchymal epithelial transition factor gene.

\section{Statistical analyses}

The statistical analyses were performed based on all screened subjects with an evaluable MET gene status assessment. Descriptive statistics for continuous variables are provided as mean, standard deviation, and median, while frequency and percent distributions were provided for non-continuous variables. To investigate $M E T$ gene status prevalence within each subgroup based on different baseline covariates, Chisquare tests were performed. $\mathrm{P}$ values generated from the analyses were mainly included as a descriptive measure suggesting comparative strength of association rather than a test of hypotheses. Accordingly, nominal $\mathrm{P}$ values are reported with no adjustment for multiplicity.

\section{Results}

The results presented in this study are based on FISH data from 1580 patients with $\mathrm{G} / \mathrm{GEJ} / \mathrm{E}$ adenocarcinoma screened for MET amplification in relation to an international multicentre phase II trial. The vast majority of the patients came from Europe and Australia (72.3\%) but patients from Asia, North America and South America were also screened for the trial (Table 1). The median age of the patient population was 62.0 years with more than $71 \%$ being males. For $62.1 \%$ of the patients, the tumor was localized in the stomach and for the remaining part of the population the tumor sites were esophagus, GEJ or metastatic.

\section{MET gene status}

Based on the screening of the 1,580 G/GEJ/E cancer patients, the prevalence rate of $M E T$ amplification was found to be $7.2 \%(\mathrm{~N}=113)$ and $8.7 \%(\mathrm{~N}=138)$ for MET deletion. The MET/CEN-7 ratio showed a wide range with ratios ranging from 0.5 up to 39.2. Differences were observed regarding geographic regions, sex and tumor site, as shown in Table 2. The Asian population had the highest percentage of patients with MET amplified tumors $(9.4 \%)$ and concurrently the lowest percentage of MET deletion $(3.2 \%)(\mathrm{P}<0.0001$, Chi-square test). With regard to sex, MET deletion was more frequent among males $(10.1 \%)$ compared to females $(5.3 \%)(\mathrm{P}=0.0082$, Chi-square test). The tumor site seems to have less influence on the prevalence of $M E T$ amplification except for the metastatic tumors where $9.1 \%$ were found to be amplified, which is slightly higher than for the other tumor sites. For the patients with MET deletion, a prevalence of $17.6 \%$ was found when the tumor was located in esophagus compared to $5.7 \%$ in the stomach $(\mathrm{P}<0.0001$, Chi-square test).

\section{MET signal distribution}

In addition to the calculation of the $M E T / C E N-7$ ratios and determination of gene status an assessment of the signal distribution pattern was performed. Data from this assessment was available from 1,579 of the 1,580 screened patients as shown in Table 3. In total, $6.3 \%$ of the screened population showed a heterogeneous signal distribution, either focal or mosaic with the mosaic distribution pattern slightly more prevalent $(4.0 \%)$ than the focal distribution $(2.3 \%)(\mathrm{P}=0.0051$, One proportion z-test). No major differences were observed regarding the distribution between the heterogeneous and the homogenous pattern when it comes to sex, tumor site, and geographic regions (Table 3). 
Table 2 MET screening results and gene status based on $M E T / C E N-7$ ratio $(\mathrm{N}=1,580)$

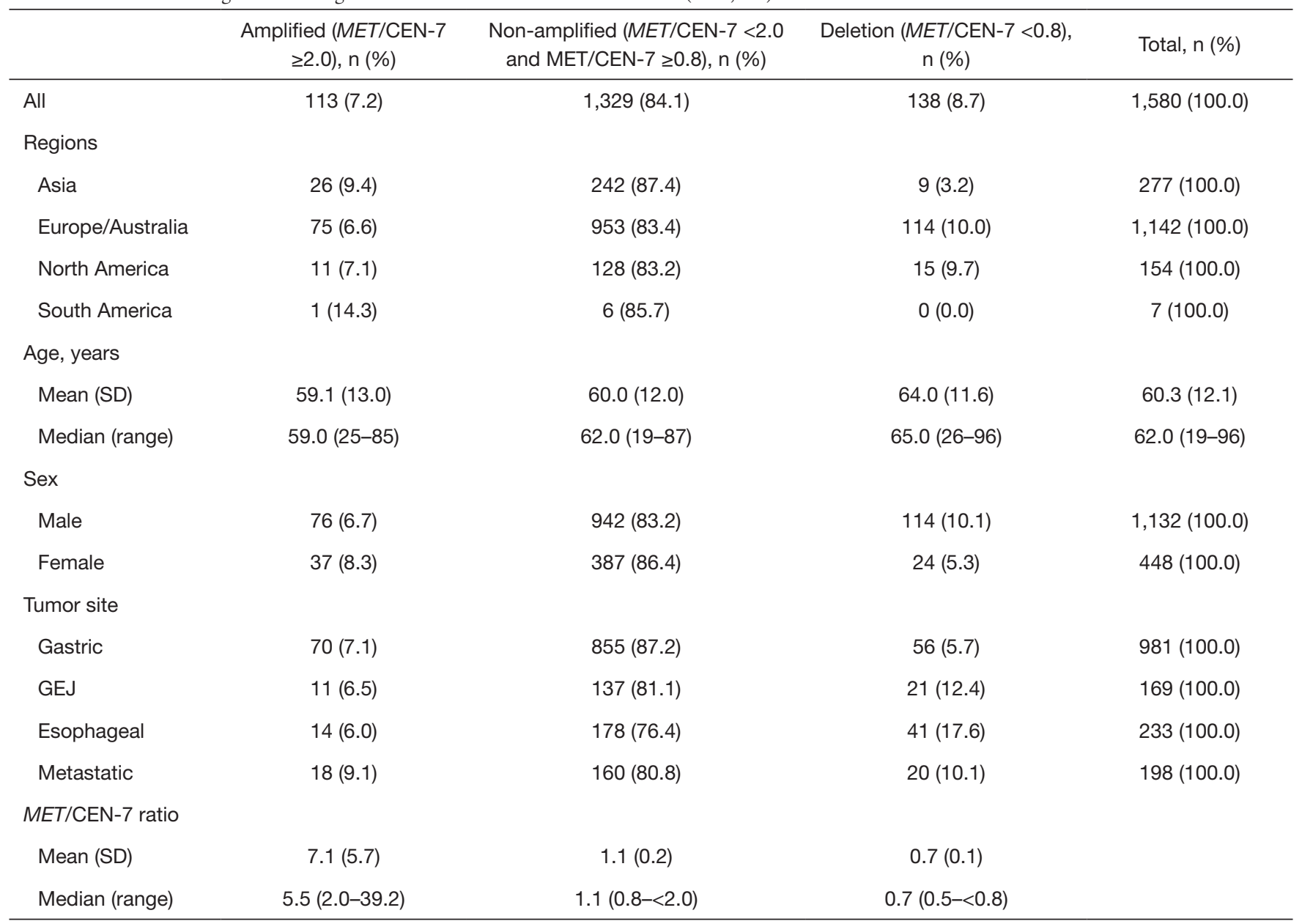

MET, mesenchymal epithelial transition factor gene. CEN-7, centromere of chromosome 7; GEJ, gastroesophageal junction.

The link between $M E T$ gene status and signal distribution is shown in Table 4. For patients with MET amplified tumors, a total of $51.3 \%$ also had a heterogeneous signal distribution. This link was especially strong for the group of patients with a focal signal distribution pattern as no patients with focal heterogenous signal distribution were found among the MET non-amplified or deleted groups, as shown in Figure 1. The mosaic heterogeneous signal distribution was detected in 64 out of the 1,579 screened patients and found among all three MET gene status categories; amplified, non-amplified and deletion. The proportion of patients who had MET amplification and a mosaic signal distribution was $34.4 \%$ (Table 4). Figure 1 visualize the distribution of the MET/CEN-7 ratios in relation to the three gene signal distribution patterns. Patients who harbored tumors with MET deletion were mainly found in the group with a homogenous signal distribution, as shown in Table 4 and Figure $1 B$.

\section{Discussion}

So far, our knowledge on $M E T$ amplification in patients with G/GEJ/E cancer has been based on data from relatively small studies that have included up to a few hundred patients (7-19). Here, we report MET gene aberration data from 1,580 patients with G/GEJ/E adenocarcinoma analyzed with a validated MET FISH assay using the formamide-free, fast IQFISH hybridization buffer. To the best of our knowledge, it is the largest cohort reported so far. Compared to other analytical methods for detection of gene aberrations, such as qPCR and nextgeneration sequencing, the ISH technology offers the 
Table 3 MET screening results and signal distribution pattern $(\mathrm{N}=1,579)^{1}$

\begin{tabular}{|c|c|c|c|c|}
\hline & Focal Heterogeneous, $\mathrm{n}(\%)$ & Mosaic Heterogeneous, n (\%) & Homogenous, n (\%) & Total, n (\%) \\
\hline \multicolumn{5}{|l|}{ Regions } \\
\hline Asia & $11(4.0)$ & $6(2.2)$ & $259(93.8)$ & $276(100.0)$ \\
\hline Europe/Australia & $23(2.0)$ & $55(4.8)$ & $1,064(93.2)$ & $1,142(100.0)$ \\
\hline South America & $0(0.0)$ & $1(14.3)$ & $6(85.7)$ & $7(100.0)$ \\
\hline \multicolumn{5}{|l|}{ Sex } \\
\hline Male & $25(2.2)$ & $45(4.0)$ & $1,061(93.8)$ & $1,131(100.0)$ \\
\hline Female & $11(2.5)$ & $19(4.2)$ & $418(93.3)$ & $448(100.0)$ \\
\hline GEJ & $3(1.8)$ & $5(3.0)$ & $161(95.3)$ & $169(100.0)$ \\
\hline Esophageal & $7(3.0)$ & $12(5.2)$ & $214(91.8)$ & $233(100.0)$ \\
\hline Metastatic & $7(3.5)$ & $7(3.5)$ & $184(93.0)$ & $198(100.0)$ \\
\hline
\end{tabular}

${ }^{1}$ Data on signal distribution was missing for one patient. MET, mesenchymal epithelial transition factor gene; GEJ, gastroesophageal junction.

Table 4 MET gene status based on MET/CEN-7 ratio and signal distribution pattern $(\mathrm{N}=1,579)^{1}$

\begin{tabular}{|c|c|c|c|c|}
\hline & $\begin{array}{l}\text { Amplified (MET/CEN-7 } \\
\quad \geq 2.0), \mathrm{n}(\%)\end{array}$ & $\begin{array}{c}\text { Non-Amplified (MET/CEN-7 } \\
<2.0 \text { and MET/CEN-7 } \geq 0.8), \mathrm{n}(\%)\end{array}$ & $\begin{array}{c}\text { Deletion (MET/CEN-7 <0.8) } \\
\mathrm{n}(\%)\end{array}$ & Total, n (\%) \\
\hline All & $113(7.2)$ & $1,328(84.1)$ & $138(8.7)$ & $1,579(100.0)$ \\
\hline \multicolumn{5}{|l|}{ Signal distribution } \\
\hline Focal heterogeneous & $36(100.0)$ & $0(0.0)$ & $0(0.0)$ & $36(100.0)$ \\
\hline
\end{tabular}

${ }^{1}$ Data on signal distribution was missing for one patient. MET, mesenchymal epithelial transition factor gene; CEN-7, centromere of chromosome 7.

possibility to study the connection between genotype and phenotype in the tumor tissues. In the current study, this option was used to investigate the link between MET gene status and the phenotypical signal distribution pattern, which has been described previously for both the HER 2 and the MET gene $(9,29)$. Determination of gene copy numbers by FISH is a well-established analysis method used for many years by pathologists and technologists to determine clinically relevant HER 2 levels in breast and gastric cancer. The current FISH ratio method in which MET loci and the centromeric region of chromosome 7 are detected using separate fluorescent labels has been thoroughly validated (9) and is completely analogous to determination of the HER2/CEN17 ratio also performed in gastric cancer tissue. Therefore, we have no reason to suspect a bias in the reported results should be introduced by the testing method.

The median age of the study population was 62.0 years with the typical sex distribution for G/GEJ/E cancer of approximately two third being males (35). Among the 1,580 screened patients, 113 cases were found to be amplified corresponding to a prevalence rate of $7.2 \%$, which is within the range of what other studies have shown using different type of ISH assays (7-15). When comparing 

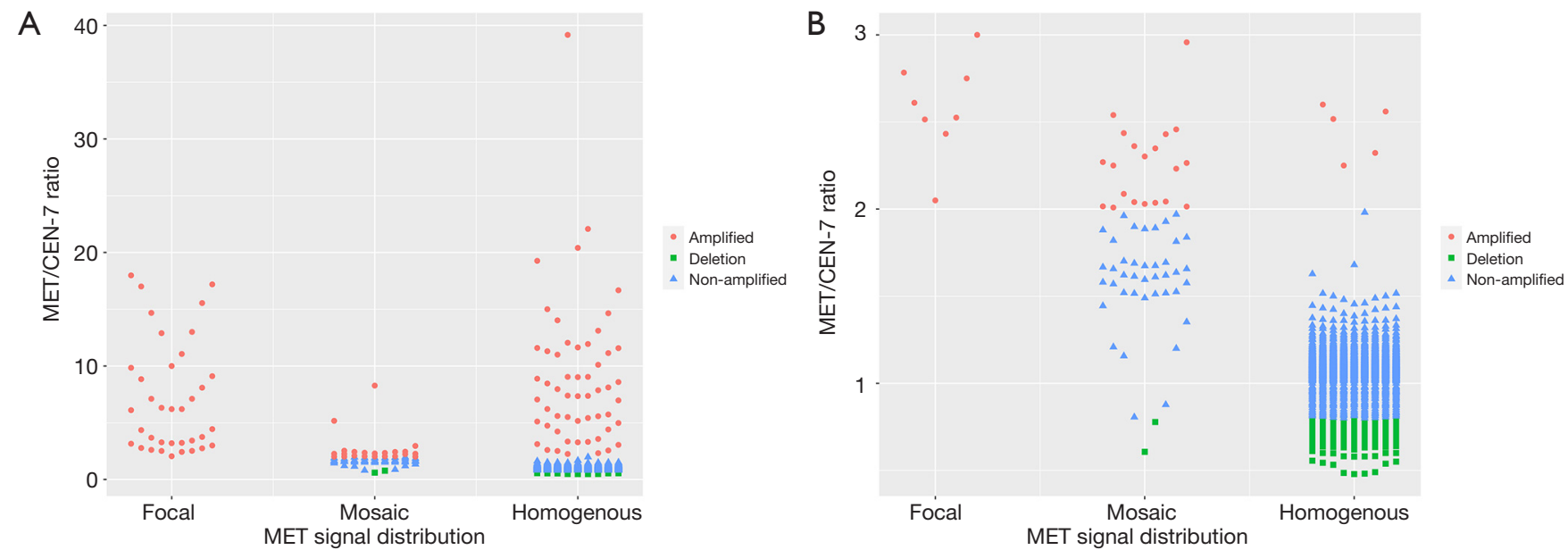

Figure 1 Dot plot of $M E T / C E N-7$ ratios versus the signal distribution pattern for all patient specimens (N=1,579) (A). Data on signal distribution is missing for one patient. Dot plot of $M E T / C E N-7$ ratios versus the signal distribution pattern for all patient specimens up to a MET/CEN-7 ratio of 3.0 (N=1,499) (B). MET, mesenchymal epithelial transition factor gene; CEN-7, centromere of chromosome 7 .

the different geographical regions, MET amplification was significantly more prevalent in the Asian patient population compared to patients screened in other regions. No published studies have investigated this population difference previously, hence, it is difficult to confirm if a region/race difference exists with respect to the prevalence rate of $M E T$ amplification in G/GEJ/E cancer patients. However, a couple of studies conducted in China have shown relatively high prevalence rates; one using a FISH assay showed a prevalence of $8.3 \%$ and another study using a qPCR assay detected MET amplification in $30.5 \%$ of the patients $(8,16)$. Again, it should be underlined that results from these two types of assays are not directly comparable but data from these studies could indicate a relatively high rate of $M E T$ amplification among Asian patients. No major difference was found in the prevalence rate for $M E T$ amplification between the three tumor sites (G/GEJ/E) except for the metastatic sites, which were slightly higher. The information in the literature on tumor site differences is limited, but a single published study with similar types of patients seems to confirm our findings (13).

A link between gene amplification and a heterogeneous signal distribution pattern was found in the study. More than $50 \%$ of the patients with $M E T$ amplified tumors had a heterogeneous gene signal distribution, either focal or mosaic. For one group of patients, this link was especially strong, as all patients having tumors with a focal signal distribution were among the 113 patients with $M E T$ amplification. The link between MET amplification and a heterogeneous signal distribution pattern was also shown when the assay was validated (9), and a similar correlation has previously been reported among patients with HER2 amplified G/GEJ/E tumors (29). It is known that Met plays a key role in the malignant transformation especially in tumors with MET amplification, leading to an invasive growth with increased cell motility and dissociation as well as tissue infiltration and stimulation of angiogenesis (36). The fact that the focal heterogeneous signal distribution is ultimately linked to MET amplification status is surprising and it could indicate that $M E T$ amplification is a requirement for the appearance of focal heterogenous signal distribution. Previous studies in adenocarcinomas of the colon have indicated that HGF secreted from stromal cells in the tumor microenvironment activates Wnt- and betacatenin signaling, which is important for maintenance of cancer stem cells and de-differentiation of non-cancer stem cells into cancer stem cells (37). Since Met signaling has been shown to enhance tumor growth, stimulate cancer cell motility and increase invasiveness, it would be interesting in future studies to determine if focal areas with MET amplified tumor cells in adenocarcinomas of G/GEJ/E are clinically relevant entities that populate cancer stem cells or highly tumorigenic cells and drives disease progression. The link between the mosaic signal distribution and gene amplification was somewhat weaker and here, approximately $35 \%$ of the patients had MET amplified tumors.

In the current study, we also investigated the prevalence rate of $M E T$ deletion, defined as $M E T / C E N-7<0.8$, and 
surprisingly, this aberration was found in 138 patients corresponding to $8.7 \%$ of the screened population. Unexpectedly, this prevalence rate was higher than for $M E T$ amplified tumors and the MET deletions were almost exclusively found among tumors with a homogeneous signal distribution pattern. Data in the literature on MET tumor deletion is very limited and we have only been able to identify a single publication on the topic. This is from a study investigating $M E T$ gene aberrations in a group of patients with salivary gland cancer using a FISH assay and here, the prevalence of $M E T$ tumor deletion was found to be $7.7 \%$ (22). Furthermore, in this study MET amplification and $M E T$ deletion were clearly associated with patient age, male gender, increased tumor size, lymph node metastasis and high-grade malignancy. The implications of $M E T$ deletion in relation to tumor growth and survival is largely unknown but based on an experimental study using a liver cancer model, it has been suggested that loss of MET signaling increases cellular stress leading to an imbalance in redox homeostasis in the hepatocytes resulting in tumor progression (38). There seems to be a greater variability for the $M E T$ deletion data compared to what has been observed for MET amplification with respect to the different study variables. A significant sex difference was shown with a nearly doubling of the MET deletion rate in males (10.1\%) compared to females (5.3\%). Furthermore, a likewise significant difference in the prevalence of MET deletions was found regarding tumor site. Here, a prevalence of $5.7 \%$ was found for the stomach, increasing to $12.4 \%$ and $17.6 \%$ for the GEJ and esophagus, respectively.

The data obtained from this screening population does not give direct mechanistic information regarding activity of Met transcripts or proteins in these tumors. Met expression in the partially MET deleted tumors could be highly active due to changes in transcriptional regulation or due to activating mutations. Therefore, additional understanding of the mechanisms affected by Met during cancer progression could be relevant for new treatment options and optimal biomarker detection. In the current study access to demographic and clinicopathological information was limited, which prevented correlation of MET gene status with this type of information. Although this is a limitation of the study, we believe that observations on the MET amplification and deletion as well as the phenotypical gene signal distribution pattern are relevant, especially as they are based on data from a large population of patients with G/GEJ/E cancer.

In summary, the current study provided the largest dataset on the prevalence of MET amplification and deletion in patients with G/GEJ/E cancer. The prevalence of $M E T$ amplification found in our population was $7.2 \%$. Furthermore, the data confirmed an association between MET amplification and the heterogeneous signal distribution previously shown in relation to the validation of the MET IQFISH assay (9). Unexpectedly, our study showed a relatively high prevalence of $M E T$ gene deletion, which has not previously been described for G/GEJ/ E cancer. A total of $8.7 \%$ of the patients were found to have tumors with $M E T$ deletion and these were almost exclusively linked to a homogeneous signal distribution. Current knowledge of the role of MET deletion in relation to tumor development is limited, but since MET deletions have been observed in context of increased malignancy it may play a role in oncogenic transformation of the cells as has been described for MET amplifications.

\section{Acknowledgments}

Funding: The study was funded by Amgen Inc., Thousand Oaks, USA.

\section{Footnote}

Reporting Checklist: The authors have completed the STREGA reporting checklist. Available at http://dx.doi. org/10.21037/atm-20-4081

Data Sharing Statement: Available at http://dx.doi. org/10.21037/atm-20-4081

Conflicts of Interest: All authors have completed the ICMJE uniform disclosure form (available at http://dx.doi. org/10.21037/atm-20-4081). JTJ reports other from Employee of Agilent Technologies, personal fees from Agilent Technologies, during the conduct of the study; personal fees from Leo Pharma, personal fees from Alligator Bioscience, personal fees from Agilent Technologies, personal fees from Oncology Venture, personal fees from Azanta, personal fees from Euro Diagnostica, outside the submitted work. He also serves as an unpaid editorial board member of Annals of Translational Medicine from Nov 2016 to Oct 2022. JM reports personal fees from employment by Agilent Technologies, outside the submitted work. The other authors have no conflicts of interest to declare.

Etbical Statement: The authors are accountable for all aspects of the work in ensuring that questions related 
to the accuracy or integrity of any part of the work are appropriately investigated and resolved. The study was conducted according to the Declaration of Helsinki (as revised in 2013), and informed consent was received from patients prior to testing of the biopsy specimens. Prior to study initiation the protocol was reviewed and approved by Institutional Review Boards/Ethics Committees in the respective countries. The study was conducted under a US Investigational New Drug Application and Investigational Device Exemption according to the International Conference on Harmonization (ICH) Good Clinical Practice (GCP) guideline. The study was conducted at 97 clinical sites worldwide. ClinicalTrials. gov ID: NCT02016534 (https://clinicaltrials.gov/ct2/ show/NCT02016534). EudraCT Number: 2013-00127724 (https://www.clinicaltrialsregister.eu/ctr-search/ search?query=2013-001277-24).

Open Access Statement: This is an Open Access article distributed in accordance with the Creative Commons Attribution-NonCommercial-NoDerivs 4.0 International License (CC BY-NC-ND 4.0), which permits the noncommercial replication and distribution of the article with the strict proviso that no changes or edits are made and the original work is properly cited (including links to both the formal publication through the relevant DOI and the license). See: https://creativecommons.org/licenses/by-nc-nd/4.0/.

\section{References}

1. Cooper CS, Park M, Blair DG, et al. Molecular cloning of a new transforming gene from a chemically transformed human cell line. Nature 1984;311:29-33.

2. Marano L, Chiari R, Fabozzi A, et al. c-Met targeting in advanced gastric cancer: An open challenge. Cancer Lett 2015;365:30-6.

3. Matsumoto K, Umitsu M, De Silva DM, et al. Hepatocyte growth factor/MET in cancer progression and biomarker discovery. Cancer Sci 2017;108:296-307.

4. Bradley CA, Salto-Tellez M, Laurent-Puig P, et al. Targeting c-MET in gastrointestinal tumours: rationale, opportunities and challenges. Nat Rev Clin Oncol 2017;14:562-76.

5. Kawakami H, Okamoto I, Okamoto W, et al. Targeting MET Amplification as a New Oncogenic Driver. Cancers (Basel) 2014;6:1540-52.

6. Kawakami H, Okamoto I. MET-targeted therapy for gastric cancer: the importance of a biomarker-based strategy. Gastric Cancer 2016;19:687-95.

7. Jardim DL, Tang C, Gagliato Dde M, et al. Analysis of 1,115 patients tested for MET amplification and therapy response in the MD Anderson Phase I Clinic. Clin Cancer Res 2014;20:6336-45.

8. An X, Wang F, Shao Q, et al. MET amplification is not rare and predicts unfavorable clinical outcomes in patients with recurrent/metastatic gastric cancer after chemotherapy. Cancer 2014;120:675-82.

9. Jørgensen JT, Nielsen KB, Mollerup J, et al. Detection of MET amplification in gastroesophageal tumor specimens using IQFISH. Ann Transl Med 2017;5:458.

10. Catenacci DV, Ang A, Liao WL, et al. MET tyrosine kinase receptor expression and amplification as prognostic biomarkers of survival in gastroesophageal adenocarcinoma. Cancer 2017;123:1061-70.

11. Ooi A, Oyama T, Nakamura R, et al. Semi-comprehensive analysis of gene amplification in gastric cancers using multiplex ligation-dependent probe amplification and fluorescence in situ hybridization. Mod Pathol 2015;28:861-71.

12. Lee HE, Kim MA, Lee HS, et al. MET in gastric carcinomas: comparison between protein expression and gene copy number and impact on clinical outcome. $\mathrm{Br} \mathrm{J}$ Cancer 2012;107:325-33.

13. Lennerz JK, Kwak EL, Ackerman A, et al. MET amplification identifies a small and aggressive subgroup of esophagogastric adenocarcinoma with evidence of responsiveness to crizotinib. J Clin Oncol 2011;29:4803-10.

14. Kawakami H, Okamoto I, Arao T, et al. MET amplification as a potential therapeutic target in gastric cancer. Oncotarget 2013;4:9-17.

15. Gavine PR, Ren Y, Han L et al. Volitinib, a potent and highly selective c-Met inhibitor, effectively blocks c-Met signaling and growth in c-MET amplified gastric cancer patient-derived tumor xenograft models. Mol Oncol 2015;9:323-33.

16. Shi J, Yao D, Liu W, et al. Frequent gene amplification predicts poor prognosis in gastric cancer. Int $\mathrm{J}$ Mol Sci 2012;13:4714-26.

17. Janbabai G, Oladi Z, Farazmandfar T, et al. The prognostic impact of EGFR, ErbB2 and MET gene amplification in human gastric carcinomas as measured by quantitative Real-Time PCR. J Cancer Res Clin Oncol 2015;141:1945-52.

18. Graziano F, Galluccio N, Lorenzini P, et al. Genetic activation of the MET pathway and prognosis of patients with high-risk, radically resected gastric cancer. J Clin 
Oncol 2011;29:4789-95.

19. Matsusaka S, Kobunai T, Yamamoto N, et al. Prognostic impact of KRAS mutant type and MET amplification in metastatic and recurrent gastric cancer patients treated with first-line S-1 plus cisplatin chemotherapy. Genes Cancer 2016;7:27-35.

20. Guo R, Luo J, Chang J, et al. MET-dependent solid tumours - molecular diagnosis and targeted therapy. Nat Rev Clin Oncol 2020;17:569-87.

21. Heydt C, Becher AK, Wagener-Ryczek S, et al. Comparison of in situ and extraction-based methods for the detection of MET amplifications in solid tumors. Comput Struct Biotechnol J 2019;17:1339-47.

22. Ach T, Zeitler K, Schwarz-Furlan S, et al. Aberrations of MET are associated with copy number gain of EGFR and loss of PTEN and predict poor outcome in patients with salivary gland cancer. Virchows Arch 2013;462:65-72.

23. Peng Z, Zhu Y, Wang Q, et al. Prognostic significance of MET amplification and expression in gastric cancer: a systematic review with meta-analysis. PLoS One 2014;9:e84502.

24. Lee J, Tran P, Klempner SJ. Targeting the MET Pathway in Gastric and Oesophageal Cancers: Refining the Optimal Approach. Clin Oncol (R Coll Radiol) 2016;28:e35-44.

25. Yasui H, Go N, Yang H, et al. A Phase 1 study evaluating AMG 337 in Asian patients with advanced solid tumors. Jpn J Clin Oncol 2017;47:772-6.

26. Van Cutsem E, Karaszewska B, Kang YK, et al. A Multicenter Phase II Study of AMG 337 in Patients with MET-Amplified Gastric/Gastroesophageal Junction/ Esophageal Adenocarcinoma and Other MET-Amplified Solid Tumors. Clin Cancer Res 2019;25:2414-23.

27. Sanchez-Vega F, Hechtman JF, Castel P, et al. EGFR and MET Amplifications Determine Response to HER2 Inhibition in ERBB2-Amplified Esophagogastric Cancer. Cancer Discov 2019;9:199-209.

28. Ebert K, Mattes J, Kunzke T, et al. MET as resistance factor for afatinib therapy and motility driver in gastric

Cite this article as: Jørgensen JT, Mollerup J, Yang H, Go N, Nielsen KB. MET deletion is a frequent event in gastric/ gastroesophageal junction/esophageal cancer: a cross-sectional analysis of gene status and signal distribution in 1,580 patients. Ann Transl Med 2021;9(3):225. doi: 10.21037/atm-20-4081 cancer cells. PLoS One 2019;14:e0223225.

29. Jørgensen JT, Nielsen KB, Kjærsgaard G, et al. HER2 Amplification and Gene Signal Distribution in Gastroesophageal Cancer. J Cancer.2017;8:1517-24.

30. Matthiesen SH, Hansen CM. Fast and non-toxic in situ hybridization without blocking of repetitive sequences. PLoS One 2012;7:e40675.

31. Viale G, Paterson J, Bloch M, et al. Analysis of HER2 status in gastroesophageal tumor specimens using a new automated HER2 IQFISH pharmDx ${ }^{\mathrm{TM}}$ (Dako Omnis) assay. Histol Histopathol 2016;31:1327-35.

32. Di Leo A, Gancberg D, Larsimont D, et al. HER-2 Amplification and Topoisomerase II $\alpha$ Gene Aberrations as Predictive Markers in Node-positive Breast Cancer Patients Randomly Treated Either with an Anthracyclinebased Therapy or with Cyclophosphamide, Methotrexate, and 5-Fluorouracil. Clin Cancer Res 2002;8:1107-16.

33. Nielsen KV, Ejlertsen B, Møller S, et al. The value of TOP2A gene copy number variation as a biomarker in breast cancer: Update of DBCG trial 89D. Acta Oncol 2008;47:725-34.

34. Vizcaíno MA, Shah S, Eberhart CG, et al. Clinicopathologic implications of NF1 gene alterations in diffuse gliomas. Hum Pathol 2015;46:1323-30.

35. Marqués-Lespier JM, González-Pons M, Cruz-Correa M. Current Perspectives on Gastric Cancer. Gastroenterol Clin North Am 2016;45:413-28.

36. Appleman LJ. MET signaling pathway: a rational target for cancer therapy. J Clin Oncol 2011;29:4837-8.

37. Vermeulen L, De Sousa E Melo F, van der Heijden M, et al. Wnt activity defines colon cancer stem cells and is regulated by the microenvironment. Nat Cell Biol 2010;12:468-76.

38. Takami T, Kaposi-Novak P, Uchida K, et al. Loss of hepatocyte growth factor/c-Met signaling pathway accelerates early stages of $\mathrm{N}$-nitrosodiethylamine induced hepatocarcinogenesis. Cancer Res 2007;67:9844-51. 